\title{
Performability Analysis of Handoff Calls in Personal Communication Networks
}

\author{
Cheul Woo Ro* \\ Dept. of Computer Science \\ Pusan Women's University \\ Pusan, Korea \\ E-mail:cwro@ee.duke.edu
}

\author{
Kishor S. Trivedi \\ Center for Advanced Computing and Communication \\ Dept. of Electrical and Computer Engineering \\ Duke University \\ Durham, NC 27708-0291 \\ kst@ee.duke.edu
}

\begin{abstract}
A combined performance and dependability(called performability) model for dealing with handoff calls is introduced. Stochastic reward nets(SRNs) are used for this purpose. An SRN model of channel assignment is developed and analyzed. The method of phase type expansion is used to approximate non-exponential call holding time distribution. A discussion of how handoff models with failure relate to Markov reward models is also given.
\end{abstract}

\section{Introduction}

The success of a proposed computer system or network depends critically on its performance, dependability (e.g., reliability and availability), and performability (performance, taking failures account) $[1,2]$. Performability evaluation seeks to determine user-oriented measures of performance, taking into account changes in performance due to failurerelated behavior. Simultaneous consideration of performance and fault related behaviors is very important in a communication system, where faults can occur that impact the system's behavior but do not cause the system to fail completely. This is a common attribute in modern distributed systems. Markov reward models (MRMs) [2] provide a natural framework for combined performance and dependability analysis of such systems. SRNs [3] is a formalism for a high level specification of MRMs.

\footnotetext{
*Supported by grants from Korea Science and Engineering Foundation, the Lord Foundation and a Duke University Research Instrumentation award.
} 
A personal commnication network (PCN) [4] is a digital network that provides high quality wireless access for personal commnication service (PCS) subscribers. Performance and performability modeling and analysis are necessary to aid the designer to ensure a high quality of PCS while minimizing costs. When a call attempt occurs in a cell in PCN, the controller (base station) carries out channel allocation. A call attempt can be served by an available channel from a channel pool with a fixed number of channels in fixed channel allocation scheme $[5,6]$, considered in this paper. We review the concept of MRMs and we show how handoff calls (channel assignment) can be modeled and analyzed with the assistance of SRNs. A new call and a handoff call compete to get a channel in a cell within the PCN and channel holding times of calls follow a general distribution. We use an approximation based on phase-type expansion to deal with such a non-Markovian model. The blocking probabilities of a new call and a handoff call are computed for the performance comparison of four handoff methods using our SRN model. The results are compared with both discrete event simulation results and Hong's method [7]. In order to capture a channel failure while serving a call, we consider different schemes. First, a new channel is reassigned to the blocked call after which the call is resumed. Second, the blocked call is lost.

The paper is organized as follows: Section 2 describes the traffic model of handoff calls, and the moments matching method for the phase type approximation. Section 3 shows how different handoff methods can be modeled by SRNs and describes how to introduce channel failure and channel reassignment in SRN models. Section 4 presents the numerical results for both performance and performability measures of interest.

\section{System Description}

In a PCN, a call originated in a cell gets a channel and holds it until that call is completed in the cell or the portable moves out of the cell. The channel holding time is either the call duration time or the time for which the portable resides in the cell whichever is less. The channel holding time follows a general distribution and hence it is difficult to compute the call blocking probabilities in a cell by a standard queueing analysis. So we propose to model the channel utilization behavior of a cell by SRN. But the SRN allows only the exponential firing time distributions, so we approximate the channel holding time using the phase type approach. 


\subsection{Traffic Model}

\subsubsection{Channel Holding Time in a Cell}

The channel holding time $T_{H}$ in a cell is defined as the time duration between the instant that a channel is occupied by a call and the instant it is released by either completion of the call or a cell boundary crossing by the portable, whichever is less. This is a function of parameters such as the cell radius $R(\mathrm{~km})$, the portable speed $V(\mathrm{~km} / \mathrm{hr})$, the direction of the portable, etc. The call duration time $T_{M}$ is assumed to be exponentially distributed with the mean $\frac{1}{\mu_{M}}$. When the velocity of a portable is constant in a cell and the direction of travel is uniformly distributed, a portable originating a new (handoff) call resides in a cell for time $T_{n}\left(T_{h}\right)$, with the following probability density [7]:

$$
\begin{gathered}
f_{T n}(t)=\frac{2 V}{\pi R^{2}} \sqrt{R^{2}-\left(\frac{V t}{2}\right)^{2}}, \quad\left(0 \leq t \leq \frac{2 R}{V}\right) \\
f_{T h}(t)=\frac{V}{\pi \sqrt{R^{2}-\left(\frac{V t}{2}\right)^{2}}}, \quad\left(0 \leq t \leq \frac{2 R}{V}\right)
\end{gathered}
$$

The channel holding times of a new call and handoff call are given by:

$$
T_{H n}=\min \left(T_{M}, T_{n}\right), T_{H h}=\min \left(T_{M}, T_{h}\right)
$$

Let the integer random variable $K$ be the number of times that a nonblocked call is successfully handed off during its lifetime. The mean value of $K$ is computed as:

$$
\bar{K}=\sum_{k=0}^{\infty} k P_{r}(K=k)=\sum_{k=0}^{\infty} k P_{N} P_{H}^{k-1}\left(1-P_{H}\right)=\frac{P_{N}}{\left(1-P_{H}\right)}
$$

, where $P_{N}=P_{r}\left(T_{M}>T_{n}\right), P_{H}=P_{r}\left(T_{M}>T_{h}\right)$.

\subsubsection{Approximating Channel Holding Time}

Since the SRN model allows only the exponential service time distributions, we approximate the channel holding time distribution using the phase type approach. The basic idea in this approach is to replace a non-exponential distribution in a model by a set of states and transitions between those states such that the holding time in each state is exponentially distributed. The main advantage is that the approach is conceptually simple and the entire range of CTMC solution methods is applicable. Its main drawbacks are the increase in state space, fitting a non-exponential distribution to a set of exponential phases and the inaccuracy of fit. One way of fitting is the moment matching method 
Table 1: Comparison with simulation

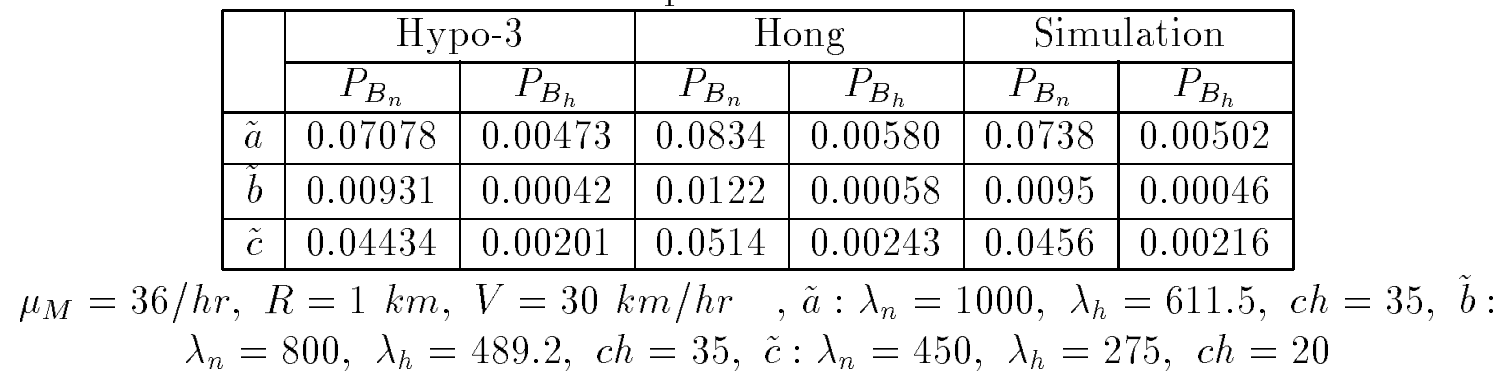

using the first, second and the third moments of channel holding times and those of the hypoexponential distribution. We obtain the moments of $T_{H}$ by numerical integration. The rates of three stages in the hypoexponential distribution can be obtained by equating the numerical values with the first three moments of the hypoexponential distribution.

We compared the accuracy of the blocking probabilities under our approximation with those obtained by Hong's method [7] and with the simulation results [11]. Table 1 shows the results. $\lambda_{n}\left(\lambda_{h}\right)$ represents new call (handoff call) arrival rate and $P_{B n}\left(P_{B h}\right)$ represents new call (handoff call) blocking probability.

From Table 1, we can see that the use of the three stage hypoexponential distributions (Hypo-3) produces results very close to discrete event simulation. So in the next section, we will approximate channel holding time by the three stage hypoexponentially distributed random variable in order to analyze the performance of the handoff methods.

\section{$2.2 \quad$ Handoff Methods}

We consider four handoff methods in order to compare their performance. These are Method 1: no priority model, Method 2: reservation channels for handoff calls, Method 3: queueing of handoff calls, and Method 4: reservation and queueing of handoff calls [5, 7].

\section{$3 \quad$ System Modeling}

\subsection{Stochastic Reward Nets}

Petri net based models have been extensively used for the performance modeling of computer and communication systems [8]. Among the Petri net based models, we 


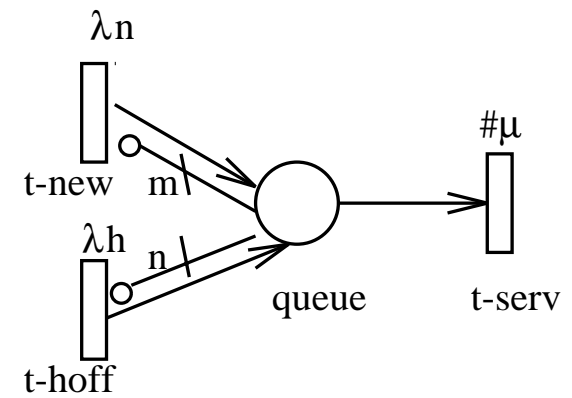

Figure 1: SRN model of the handoff methods

consider Stochastic Reward Nets (SRNs). SRNs extend the GSPN [8]. It includes all the features of GSPN and guards, timed transition priorities, variable cardinality arcs, halting condition, and reward rates. SRN model can be converted to a MRM and hence it allows calculation of reward-based measures and permits a much more concise description of system model than GSPNs do [2]. Stochastic Petri nets Package (SPNP) [9] provides support for specifying the SRN using a "C" like language and allows for the modeler to do steady state, transient, cumulative transient, upto absorption and sensitivity analysis. A graphical user interface is now available [12].

\subsection{Performance Modeling}

\subsubsection{SRN model: exponential case}

The four handoff methods can be easily modeled by SRNs as shown in Figure 1, where $N, h$, and $m$ represent total number of channels, number of reserved channels and queue size, respectively. The multiple inhibitor arc from place queue to transition $t$-new (t-hoff) means that $t$-new (t-hoff) is disabled if the number of tokens already present in the queue is $m(n)$, where value of $m(n)$ is same as the value of ena $(\mathrm{X})$ column in table 2. Tokens in queue represent new calls and handoff calls which hold the channels. If there are $\mathrm{m}$ (n) tokens in queue, no more new (handoff) calls can arrive. These tokens are released after firing transition $t$-serv, which represents the service completion of a call. The firing rate of the timed transition $t$-serv depends on the number of tokens in the queue. This is indicated by the pound symbol, $\sharp$, next to the transition.

\subsubsection{SRN model: non-exponential case}

In Figure 2, the timed transitions with respective firing rates $\mu_{1}, \mu_{2}, \mu_{3}$ represent the 3 stages of the hypoexponential distribution. They fire depending on the present service 


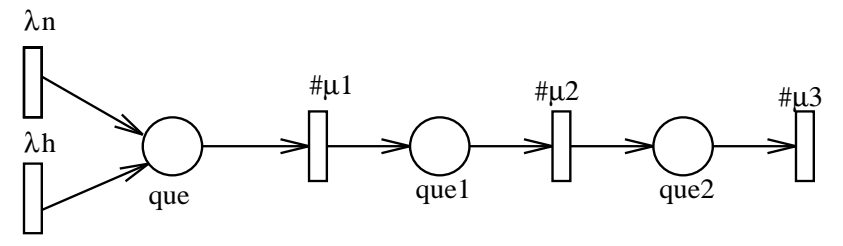

Figure 2: SRN model of the channel holding time

Table 2: Enabling function

if $(\sharp(q u e)+\sharp(q u e 1)+\sharp(q u e 2)<\operatorname{ena}(X)) \operatorname{return}(1)$; else $\operatorname{return}(0)$;

\begin{tabular}{|c|c|c|}
\hline method & transition & ena(X) \\
\hline 1 & $\lambda_{n}$ & $\mathrm{~N}$ \\
& $\lambda_{h}$ & $\mathrm{~N}$ \\
\hline 2 & $\lambda_{n}$ & $\mathrm{~N}-h$ \\
& $\lambda_{h}$ & $\mathrm{~N}$ \\
\hline 3 & $\lambda_{n}$ & $\mathrm{~N}$ \\
& $\lambda_{h}$ & $\mathrm{~N}+m$ \\
\hline 4 & $\lambda_{n}$ & $\mathrm{~N}-h$ \\
& $\lambda_{h}$ & $\mathrm{~N}+m$ \\
\hline
\end{tabular}

phase of the channel holding time. Four handoff methods can be represented using enabling functions for the transitions t-new and t-hoff instead of inhibitor arcs in SRNs as shown in Table 2. $\sharp(\mathrm{P})$ represents number of tokens in place P. Enabling functions make transitions enabled based on some explicitly stated conditions and not just on distribution of tokens in input places.

\subsection{Performability Modeling}

We extend handoff model in Figure 2 to models in Figures 3 and 4 by adding failure transitions. When a portable enters a tunnel or a subway during the conversation, the radio channels in use can fail. We consider two policy choices for the controller. First is channel reassignment and call resume scheme. After the controller detects a channel failure, it reassigns a new channel by taking one from a channel pool and updates the status of the old failed channel as available. The failed call is resumed after channel reassignment. Channel reassignment consists in switching calls already in progress from channels that these calls are using to other channels. If there is no available channel, the channel reassignment can not be performed and the call is lost. If all the channels have failed in the channel pool, the system is down. Second policy is no resume scheme. In this scheme, the controller detects channel failure and updates the status of the failed 


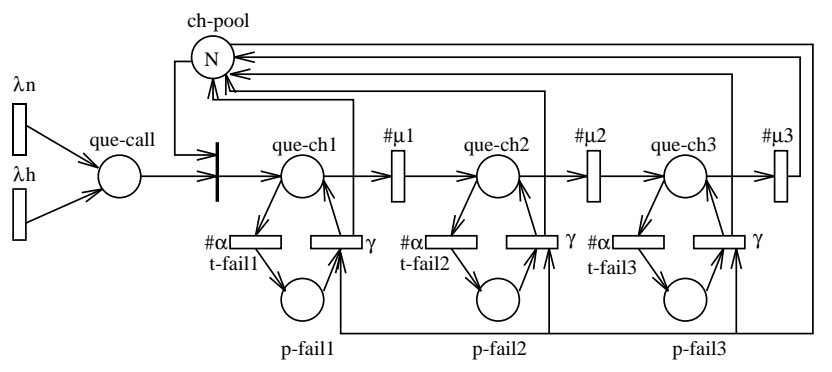

Figure 3: SRN model of handoff reliability

channel after a certain delay. The failed call is then lost. We do not consider a failure of channel when it is not in use.

\subsubsection{Channel reassignment and call resume}

Figure 3 shows the SRN model of the channel failure with resume scheme. This model can have absorbing markings if no channels are available for reassignment in a channel pool. Analysis of this model yields system reliability. The place $c h$-pool represents channel pool which initially has $N$ tokens, representing the fixed number of channels in a cell. The timed transitions with firing rates $\lambda_{n}$ and $\lambda_{h}$ are disabled if there are less than $\theta$ and $h$ tokens respectively in ch-pool. A token in the place que-call represents a call. One channel is assigned for this call by taking one token from the ch-pool. The immediate transition fires and removes one token each from que-call and ch-pool and put one token in quech1. The token in que-ch1 then moves through a sequence of exponentially distributed transitions, the combination of which represents the channel holding time. When the call is finished, the transition with firing rate $\mu 3$ fires, the channel token moves back to the ch-pool and the token for call is cleared. While the call is in service, the channel can fail. The token then moves from the que-ch $1(2,3)$ through transition $t$-faill $(2,3)$ to the place pfaill $(2,3)$, which is a place used to wait for channel reassignment and call resume. Multiple channels are competing to fail and this is depicted by appending the character $\sharp$ the rate $\alpha$. The transition with firing rate $\gamma$ reflects the assumption that one controller carries out channel reassignment. The failure rate for each channel is $\alpha$ and $\gamma$ represents the inverse of the sum of both switching and channel reassignment time.

\subsubsection{Non-resume Modeling}

Figure 4 shows channel failure model without resume. This SRN model also uses a marking dependent firing rate in a similar fashion to Figure 3 . If channel fails, the token moves from que-ch $1(2,3)$ to $p$-fail. The token then waits in this place for the updating 


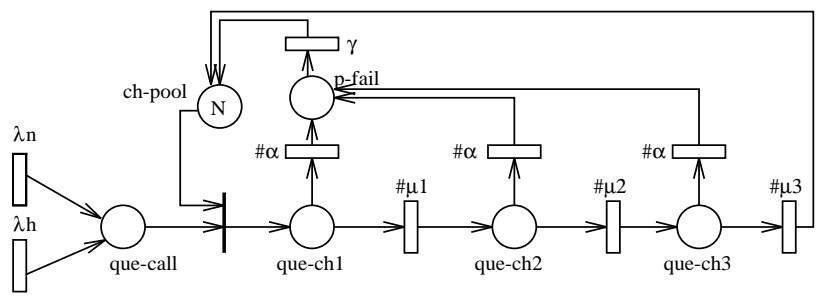

Figure 4: SRN model of handoff availability

of channel status. After that, the channel is released and put back in the ch-pool. In this model, there is no absorbing marking and analysis of this model will yield system availability.

\section{$4 \quad$ Numerical Results}

For numerical solution of our models we use SPNP with the following input parameters. - the maximum number of available channels is $N$ and fixed.

- From (4), we get $\bar{K}=0.6115$ with $V=30 \mathrm{~km} / \mathrm{hr}, \mathrm{R}=1 \mathrm{~km}$, and $\mu_{M}=36 / \mathrm{hr}$.

- the new (handoff)call arrival process is Poisson with arrival rates $\lambda_{n}\left(\lambda_{h}\right)=1000$ (611.5) calls/hour. $\lambda_{h}$ is given by $\bar{K} \lambda_{n}=0.6115 * 1000$.

- number of reserved channels $h=1,2, . .7$ and queue length parameter $m=1,2, . .7$.

- channel holding time: 3 stage hypoexponential with rates: $\mu_{1}=395.0, \mu_{2}=18750.0, \mu_{3}=$ 68.06 (in the 2 stage case for reliability, rates: $\mu_{1}=385.0, \mu_{2}=68.2473$ ).

- channel failure (reassignment) rate: $\alpha=2(\gamma=50)$ per hour.

We computed the performance measures with and without failures, which are blocking probabilities $P_{B n}$ and $P_{B h}$ for new and handoff calls, respectively, the rate at which calls are rejected, channel utilization, and system throughput. We then computed system reliability at time $t$ and system availability at time $t$. These measures are expressed in terms of the expected values of reward rate functions for an SRN.

- blocking probability: $P_{B n}$ for handoff method 1 is obtained as 0.33964 and it is the same as $P_{B h}$ when $N=20$. Figure 5 and 6 show $P_{B h}$ in the case without and with failure respectively, as a function of $h$ for Method 2 (and 4 ) and $m$ for Method 3 , $m(=1)$ is fixed for Method 4 . Figure 7 and 8 show $P_{B n}$. As expected, the values of $P_{B n}$ and $P_{B h}$ in the case with failures are higher than those with no failures. These figures demonstrate that increasing $h$ decreases $P_{B h}$. However, since the reserved channels can not be assigned to new calls, increasing $h$ significantly increases $P_{B n}$.

- rate of rejected calls: Since blocking of a handoff call means a disconnection in the middle of a call, it should be avoided as much as possible. In order to combine 


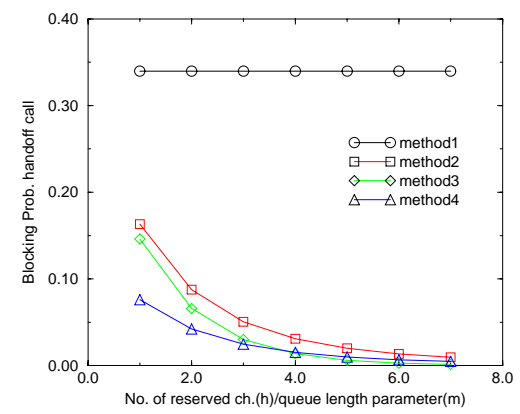

Figure 5: Blocking Probability of handoff calls without failure

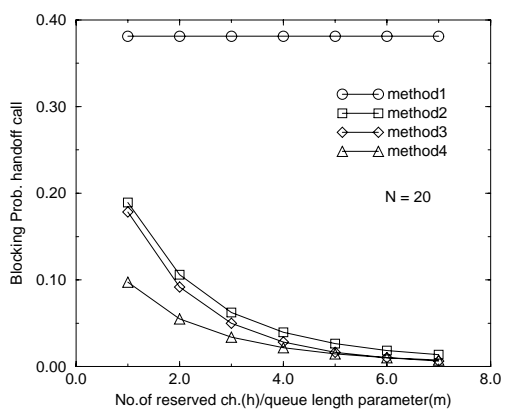

Figure 6: Blocking Probability of handoff calls with failure

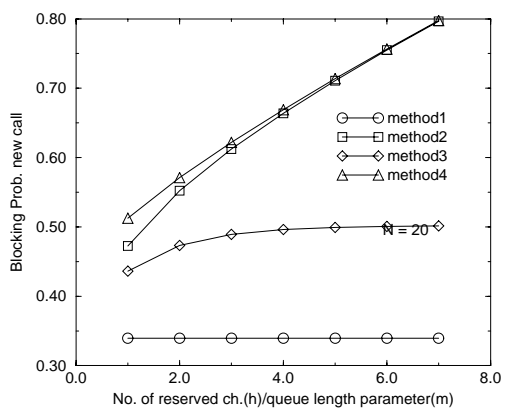

Figure 7: Blocking Probability of new calls without failure 


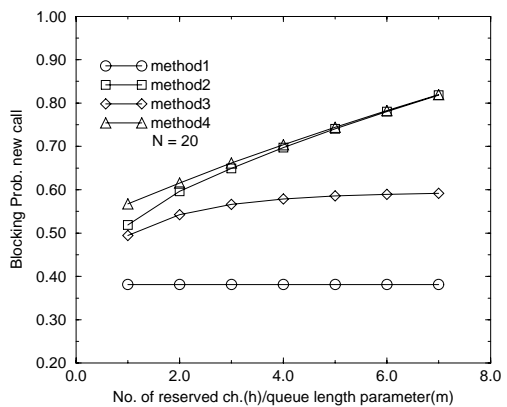

Figure 8: Blocking Probability of new calls with failure

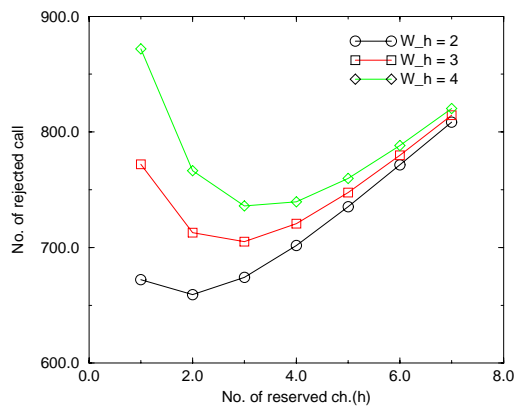

Figure 9: rate of rejected calls 


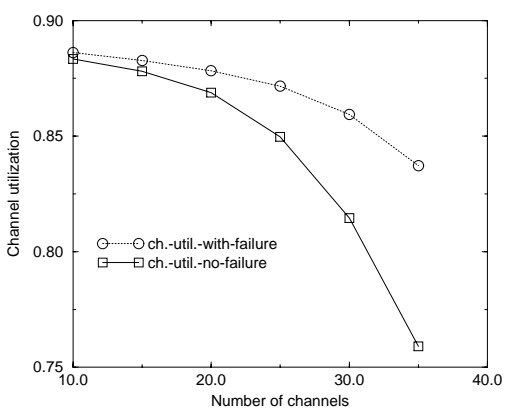

Figure 10: channel utilization

the calls rejected due to handoff and new calls we use weighting factor $W_{n}\left(W_{h}\right)$ to get the overall rate of rejected calls $\left(R_{r e j}\right)$ by assigning weighting factor $W_{n}\left(W_{h}\right)$ to $P_{B n}\left(P_{B h}\right) . R_{r e j}$ is given by $W_{n} * \lambda_{n} * P_{B n}+W_{h} * \lambda_{h} * P_{B h}$. Figure 9 shows rate of rejected calls and optimum vaules of $h$ (We vary $W_{h}$ while fixing $W_{n}=1$ ).

- channel utilization and throughput: The results of channel utilization and throughput are depicted in Figures 10 and 11 as functions of the number of channels $(N)$. These results are based on handoff Method 2 with $h=2$. As expected, the throughput without failure is higher than that with channel failure. The gap between two curves denotes the expected number of failed calls per hour, due to channel failure. Whenever the channel fails, the controller takes another channel and assigns it. This is the reason why the values of channel utilization with failure are higher than those without failure.

- reliability: Figure 12 shows system reliability as a function of time. In order to cut down the size of the underlying MRM, we had to use the 2-stage hypoexponetial model instead of 3 -stage model when $N=10$ and handoff Method $2(h=2)$.

- unavailability: Numerical results of the model's transient behavior in Figure 4 yield the instantaneous unavailability curve shown in Figure 13 as a function of time when $N=7$. As expected, these values increase with time and reach steady state values of $0.000000290462,0.0 .000000563773,0.000000477097$ for Method 2 $(h=2), 3(m=1)$, and $4(h=2, m=1)$, respectively.

\section{Conclusion}

In this paper, we showed how SRNs can be applied in the performability analysis of handoff calls in a cell. These models represented the channel utilization behavior of a 


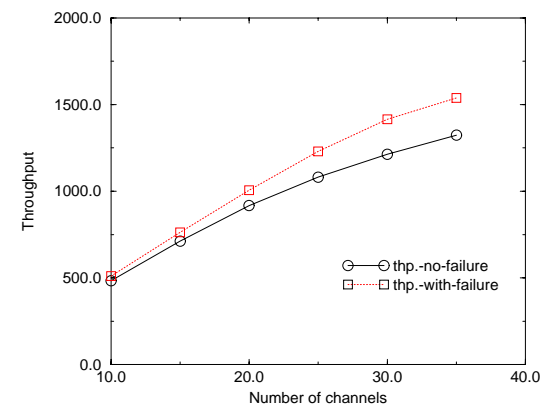

Figure 11: throughput

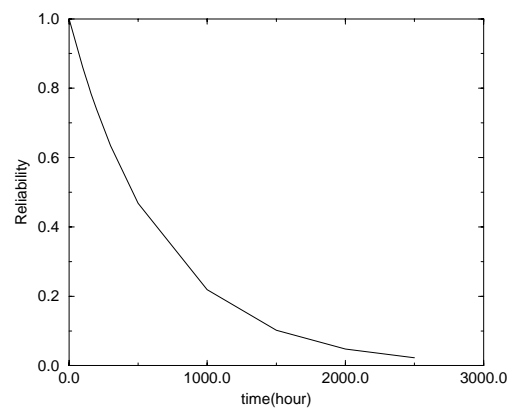

Figure 12: sytem reliability of handoff call

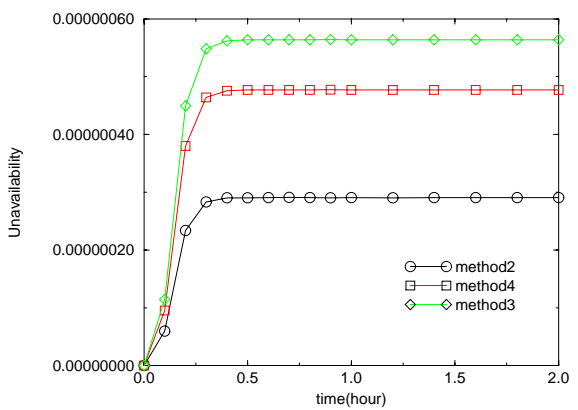

Figure 13: sytem unavailability of handoff call 
cell. In modeling with SRNs, the assumption that the transition firing times have exponential distributions is a major constraint. Since the channel holding time follows general distribution, we solved this non-exponential model using phase approximation approach. Three parameters of a hypoexponential distribution were obtained by the moment matching method. We showed how SRN models can handle four handoff methods using multiple inhibitor arcs or enabling functions and compared their performance. Method 2 and 3 achieve a lower blocking probability for handoff calls than for new call attempts, thereby providing better service quality. This is mainly due to the use of reservation channels and queueing of handoff calls.

We extend the handoff performance model to performance in the presence of failure. These performability models show the reliability and availability of handoff calls in a cell. We also evaluated combined measures of performance and reliability.

\section{References}

[1] J.F. Meyer, "Performability: A retrospective and some pointers to the future", Performance Evaluation, Vol. 14, No.3-4, February 1992, pp. 157-196.

[2] K.S. Trivedi, J.K. Muppala, S.P. Woolet, B.R. Haverkort, "Composite performance and dependability analysis", Performance Evaluation, Vol. 14, No.3-4, February 1992, pp. 197-215.

[3] G. Ciardo, A. Blakemore, P.F. Chimento, J.K. Muppala and K.S. Trivedi, "Automated generation and analysis of Markov reward models using stochastic reward nets", In Linear Algebra, Markov Chains, and Queueing Models, Carl Meyer and R.J. Plemmons (eds.), IMA Volumes in Mathematics \& its Applications, Vol. 48, pp 145-191, Springer Verlag, Heidelberg, 1993.

[4] D.C. Cox, "Personal communications- a viewpoint", IEEE Commun. Mag., Vol. 128,1990

[5] Yi-Bing Lin, "PCS Channel Assignment Strategies for Hand-off and Initial Acess", IEEE Personal Communications, Vol.1, No.3, Third Quarter, 1994.

[6] S.S. Rappaport, "The multiple-call hand-off problem in high-capacity cellular communication systems", IEEE Trans Vech Tech., Vol 40, No 3, pp 546-557, Aug 1991.

[7] D.Hong and S.S. Rappaport, "Traffic Model and Performance Analysis for Cellular Mobile Radio Telephone Systems with Prioritized and Nonprioritized Handoff Procedures", IEEE Trans. on Veh. Tech. Vol.vt35, No3. pp.77-92, August 1986. 
[8] M. Ajmone Marsan, G. Conte, and G. Balbo. "A class of Generalized Stochastic Petri Nets for the performance evaluation of multiprocessor systems," ACM Transactions on Computer Systems, Vol. 2, No. 2, pp. 93-122, 1984.

[9] G.Ciardo, K.S.Trivedi, "Manual for the SPNP Package version 3.1", Duke univ., Mar., 1992.

[10] S.C. Bruell, P.Z. Chen, and G. Balbo, "Alternative methods for incorporating nonexponential distributions into stochastic timed Petri nets," In Proc. of the 3rd Int $\%$ Workshop on Petri Nets and Performance Models (PNPM89), Kyoto, Japan, 1989.

[11] Cheul W. Ro, "Performance Analysis of Telecommunication Protocols using Stochastic Petri nets", PhD Thesis, 1994 August: Sogang Univ.

[12] R.Fricks, K.S. Trivedi. "The Development of an Integrated Modeling Environment", To appear in the Proceeding of the 1st World Congress on Systems Simul ation WCSS'97, Singapore, Sep., 1997 